\title{
L'Ellipse modale en français : arguments pour une ellipse du TP
}

\author{
Anne Dagnac \\ Université Toulouse-2-Le Mirail/ CLLE-ERSS (UMR5236) \\ dagnac@univ-tlse2.fr
}

\section{Introduction}

Contrairement à l'anglais, le français, comme d'autres langues (Italien, Espagnol, Néerlandais, Allemand entre autres), est réputé ne pas autoriser l'ellipse du syntagme verbal (Lobeck: 1995). En anglais, les auxiliaires simples (do, be, have) ou modaux (can, must,...) peuvent en effet, dans des conditions discursives adéquates, être utilisés 'nus', comme en (1) et (2) respectivement. Dans ce cas, ils sont suivis d'un syntagme verbal (désormais VP) 'vide', interprété en parallèle avec un VP antécédent, souligné dans les exemples :

(1) Peter ate a cake and John did_ too.

(2) John can talk to Peter and Mary can_ too.

La construction illustrée ci-dessus est classiquement nommée ellipse du syntagme verbal (par la suite : ellipse du VP). La nature syntaxique exacte de ce phénomène a été largement débattue depuis les années 1970 dans le cadre de la grammaire générative ${ }^{1}$. Deux approches majeures ont été avancées. Pour l'une, dite anaphore à complément nul, l'auxiliaire est syntaxiquement suivi d'une proforme nulle, notée $\varnothing$, insérée directement dans la construction syntaxique (cf., avec des variantes, Shopen : 1972, Hardt : 1999, Fiengo\&May: 1994, Chung\&al. 1995, parmi d'autres.). Elle est l'équivalent d'un pronom, et son interprétation relève de celle des constituants anaphoriques. Pour l'autre (cf., inter alia, Hankamer \& Sag 1976, Johnson 2001, Merchant 2001), désormais « analyse par effacement », ou ellipse proprement dite, la structure syntaxique contient un VP classique, parallèle à son antécédent ${ }^{2}$ qui est tardivement " effacé » lorsque la structure syntaxique est traitée par le module phonologique: le VP est de ce fait syntaxiquement présent et articulé mais phonologiquement non réalisé. Les enjeux de ce débat sont multiples, nous ne les aborderons pas tous ici. Pour les exemples anglais ci-dessus, nous adopterons l'analyse par ellipse, réservant la notion d'anaphore nulle aux exemples de type (3), dont il est admis depuis Hankamer et Sag (1976) qu'elles impliquent une proforme nulle.

(3) I asked Bill to leave but he refused. (J'ai demandé à Bill de partir mais il a refusé)

Le point commun entre le français et les autres langues citées ci-dessus est qu'aucune ne permet d'ellipse du VP avec ses auxiliaires de temps, d'aspect et de voix. Les équivalents de (1) y sont donc agrammaticaux, comme le montre (4) pour le français. Le français recourt dans ce cas à un autre type de construction, traditionnellement appelé anaphore verbale, illustrée en (5), ou à d'autres procédés.

(4) *Paul a mangé un gâteau et Pierre a aussi_. [ [mangé un gâteau]

(5) Lundi, Paul a repeint sa cuisine, et mardi, c'est Paul qui l'a fait.

Néanmoins, ces langues présentent toutes, à des degrés divers, un type de construction superficiellement comparable: on la trouve après leurs 'semi-auxiliaires' modaux pouvoir et devoir dans leurs interprétations non épistémiques, qui sont considérés comme des $\mathrm{V}$ à montée du sujet (Wurmbrand : 1999, 2001). Ce fait est illustré en (6) et (7) pour le français et l'espagnol ${ }^{3}$.

(6) Jean peut parler à Pierre et Marie peut aussi . [= 'parler à Pierre']

(7) Juan puede hablar con Pedro y Concha tambien puede. 
De façon surprenante, l'analyse de ces structures convergentes a donné lieu à des analyses opposées selon les langues. Pour l'espagnol (Depiante : 2001), l'italien (Depiante 2001, Cecchetto \& Percus : 2006) et le néerlandais (Aelbrecht : 2007a), il a été proposé que le modal soit suivi d'une proforme nulle, sans structure syntaxique interne. Pour le français en revanche, Busquets et Denis (2001) concluent qu'il s'agit d'une ellipse du VP, qu'ils baptisent «ellipse modale » : le modal y est suivi d'un VP syntaxiquement structuré, non réalisé en forme phonologique.

Précisant et complétant l'analyse de Busquets et Denis (2001), nous montrons ici que leurs conclusions sont, en grande partie, valides pour le français : il s'agit d'une ellipse. Néanmoins, les propriétés de l'ellipse modale du français divergent sur plusieurs points des ellipses du VP correspondantes en anglais. Pour en rendre compte, nous faisons l'hypothèse qu'il s'agit non pas d'une ellipse du VP, comme proposé par Busquets et Denis, mais de l'ellipse d'une proposition (désormais TP). Nous montrons également que ces ellipses sont, dans les structures impliquant une relativisation, soumises à une contrainte jusque là passée inaperçue, que nous appelons 'contrainte des sujets similaires': elle pourrait expliquer la divergence des analyses avancées pour les autres langues, et les remettre en question. Nous montrons que considérer l'ellipse modale comme une ellipse du TP offre une voie pour rendre compte de cette contrainte.

\section{Arguments pour une ellipse modale en français}

L'analyse en proforme nulle et l'analyse en ellipse s'opposent par le degré de structuration syntaxique qu'elles attribuent à l'élément manquant (désormais : la lacune). Poser une proforme nulle revient à considérer que la lacune est non syntaxiquement structurée. En conséquence, aucune opération syntaxique n'est, a priori, susceptible d'affecter un de ses sous-composants... puisqu'elle n'en a pas. En revanche, dans l'approche par ellipse, la lacune est syntaxiquement structurée, donc des opérations syntaxiques prenant place avant le traitement par le module phonologique, comme le mouvement ou le liage, peuvent cibler des sous-composants au même titre que dans la phrase non-élidée. La possibilité ou non d'extraire des éléments enchâssés dans le VP apparemment absent est donc au cœur des analyses précédentes. Pour le français, Busquets et Denis (2001) montrent que deux phénomènes, les constructions ACD et la mise en relief d'un élément en tête de phrase, traditionnellement analysés en terme de mouvement dans un cadre génératif classique (ou de dépendance à distance dans des cadres concurrents), sont possibles avec l'ellipse modale : ils justifient une analyse en ellipse, et disqualifient une analyse en proforme nulle. Dans les sous-sections suivantes, nous approfondissons les constatations de Busquets et Denis, puis, nous complétons leur argument en montrant que deux autres types de phénomènes, un mouvement- $\mathrm{A}$ et le mouvement WH-, confirment leurs arguments.

\subsection{Constructions ACD et relativisation}

Dans ces constructions (dites ACD pour Antecedent Contained Deletion), l'ellipse apparait dans une relative restrictive dont l'antécédent est dans la portée d'un quantifieur fort ou d'un défini, cf. (8) :

(8) Marie lit tous les livres qu'elle peut.

Busquets et Denis passent sous silence l'analyse syntaxique de ces relatives, qui conforte cependant leur hypothèse. En effet, la structure des relatives fait l'objet de nombreux débats, mais parmi les propositions avancées, on peut en retenir deux principales (pour une discussion et une analyse plus fine de ces deux types d'analyse, voir par exemple Hulsey et Sauerland : 2003). La première correspond à l'analyse classique d'un mouvement de l'opérateur ${ }^{4}$, où l'on a, grossièrement, la structure (9):

(9) Marie lit tous les livres [OPi qu'elle peut_<lire ti $>]^{5}$

Dans ce cas, le constituant élidé doit contenir la trace de l'opérateur, donc une position d'objet direct, ce qui plaide pour un constituant structuré. L'analyse alternative 'à tête interne' (cf. par exemple Vergnaud 1973, Kayne 1994, Bianchi 1995, Cinque 2007) ne modifie pas la ligne générale d'argumentation. C'est celle que nous retiendrons dans la suite de cette étude (cf. section 4). Si la structure de la relative est du 
type illustré en (10), «l'antécédent» est en fait inséré dans la position d'objet de lire, avant de se déplacer en spec, CP. La position d'objet de l'infinitif est donc tout aussi nécessaire, ce qui plaide pour une analyse en ellipse.

(10) Marie lit tous [DP les [spec,CP [livres $\left.]_{1}\right]$ [CP qu' elle peut $<$ tire $t_{+}>$]]

Cette conclusion, comme le notent Busquets et Denis, est confirmée par l'impossibilité d'avoir dans ce cas une proforme pleine, ce qui s'explique si le mouvement exige une position de base indisponible aussi bien avec la proforme nulle non structurée qu'avec la proforme pleine :

(11) a- * Marie lit tous les livres qu'elle le peut.

b- * Marie parle à tous les voisins qu'elle le peut.

(12) a- * Marie lit tous les livres qu'elle peut le faire.

b- * Marie parle à tous les voisins qu'elle peut le faire.

A quoi on peut ajouter que les verbes admettant un complément nul l'excluent aussi :

(13) a- *Marie a finalement transporté tous les cartons qu'elle avait d'abord refusé $\varnothing$.

b- Marie a finalement parlé à tous les voisins à qui/qu'elle avait d'abord refusé $\varnothing$.

\subsection{Mise en relief d'un constituant et « pseudo-gapping »}

Busquets et Denis notent également que l'ellipse modale permet des constructions de type pseudogapping, c'est-à-dire des ellipses du VP dont un constituant, par exemple le DP objet, a été préalablement extrait, cf. (14) :

(14) Alice ne peut pas s'acheter des jouets, mais des livres, elle peut. (B\&D : [41])

Ils ne s'attardent néanmoins pas sur les phénomènes syntaxiques en jeu dans cette construction, dont la pertinence pour trancher entre ellipse et proforme nulle n'est pas assurée.

Tout d'abord, notons que le parallèle avec le pseudo-gapping anglais n'est pas total. En effet , contrairement à ce qui se passe en anglais (comparer (14) et (15)), le constituant extrait du site élidé, ici des livres, est nécessairement placé en tête de phrase :

(14) Some brought roses and other did lilies.

'Certains ont apporté des roses et d'autres Aux des lis'

(15) *Alice ne peut pas s'acheter des jouets, mais elle peut des livres.

Pour le français, ce fait pourrait être convergent avec l'analyse du pseudo-gapping proposée par Gengel (2007), qui considère que l'objet est soumis à un «mouvement de focus » (focus movement); la construction en (14) serait ainsi le résultat d'un déplacement ouvert de l'objet dans une position focale ${ }^{6}$ en tête de phrase avant ellipse. Elle confirmerait ainsi, pour l'objet, la nécessité d'une position initiale à l'intérieur du site élidé qui, là encore, suppose une articulation syntaxique. Cet argument est néanmoins problématique. En effet, la nature de la construction en jeu est loin d'être claire. Deux analyses au moins sont possibles :

a) il s'agit effectivement d'une antéposition de focus (focus fronting), de type topicalisation, avec la structure suivante :

(16) A la rigueur, je peux lire le Figaro, mais Minute $_{\mathrm{i}}$, je ne peux pas. $<$ lire. $\mathrm{t}_{\mathrm{i}}>$

(17) J'ai pu aller à San Francisco, mais à Los Angelesi, je n'ai pas pu. < aller ti>

(18) J'ai voté pour Chirac, mais pour Sarkozy $\mathrm{j}_{\mathrm{i}}$ je ne peux pas. $<\mathrm{t}_{\mathrm{i}}>$

(19) Elle peut dire la vérité à Jules, mais à $\mathrm{Jim}_{\mathrm{i}}$, elle ne peut pas. < dire la vérité $\mathrm{t}_{\mathrm{i}}>$

Pour (16) et (17), cette analyse se heurte au fait que la phrase correspondante paraît peu plausible dans sa version non élidée : 
(16') ??A la rigueur, je peux lire le Figaro, mais Minute $\mathrm{i}_{\mathrm{i}}$ je ne peux pas lire $\mathrm{t}_{\mathrm{i}}$.

(17') ??J'ai pu aller à San Francisco, mais à Los Angeles ${ }_{i}$, je n'ai pas pu aller $\mathrm{t}_{\mathrm{i}}$.

Pour la maintenir, il faudrait supposer que la topicalisation, comme d'autres types de mouvement (cf. Merchant : 2006) est nourri par l'ellipse.

Quant à (18) et (19), ils peuvent plus directement être vus comme une topicalisation - (18') et (19') sont naturels - mais il n'est pas certain qu'ils puissent servir d'argument pour discriminer ellipse et proforme nulle. En effet, les proformes verbales pleines en le faire ont la propriété de cibler aussi bien le VP minimal (verbe et argument direct) que le VP maximal (verbe et l'ensemble des compléments de verbe). A ce titre, elles tolèrent la topicalisation des compléments indirects, cf. (19')).

(18') ?J'ai voté pour Chirac, mais pour Sarkozy $\mathrm{i}_{\mathrm{i}}$ je ne peux pas voter $\mathrm{t}_{\mathrm{i}}$.

(19’) Elle peut dire la vérité à Jules, mais à $\mathrm{Jim}_{\mathrm{i}}$, elle ne peut pas dire la vérité $\mathrm{t}_{\mathrm{i}}$

(19') ?Elle a dit la vérité à Jules, mais à $\mathrm{Jim}_{\mathrm{i}}$, elle ne peut pas le faire.

b) Une autre possibilité est de voir dans ces structures des dislocations à gauche, au moins pour les cas comme (16), sur le modèle de (16") :

(16"') A la rigueur, je peux lire le Figaro, mais Minute ${ }_{i}$, je ne peux pas $<$ te lire $>$

Cette analyse affaiblit potentiellement l'argument de Busquets et Denis. En effet, les dislocations présentant une certaine insensibilité aux contraintes d'île ${ }^{7}$, il a été proposé qu'elles ne reposent pas sur un mouvement du constituant marqué par le focus mais sur l'insertion directe en tête de phrase du constituant focalisé, associé à un pronom résomptif en position argumentale. Le mouvement ne peut donc plus être invoqué pour justifier la présence d'une position syntaxique interne à la lacune. Néanmoins, leur argument pourrait être maintenu en adoptant pour ces structures l'une des deux analyses suivantes. Dans un cas, on considère que le pronom résomptif est lié (sans mouvement) par l'élément détaché, ce qui semble cohérent avec l'interprétation de ()$^{8}:$ le liage doit « voir » à l'intérieur de la lacune.

\section{(20) [Chaque enfant] i, la maîtresse $l_{i}$ 'a félicité}

Dans l'autre, on suit Guilliot (2006) pour qui le pronom résomptif a en fait la structure [ ${ }_{\mathrm{DP}}$ le [ $\left.\mathrm{NP}_{\mathrm{N}} \ldots \mathrm{N}_{\mathrm{i}}\right]$ ], où NP est coïndicé avec le constituant focalisé, et subit une ellipse du NP, cf. $(21)^{9}$ :

(21) Je peux lire son roman, mais ses poèmes, je ne peux pas [DP les [pøèmes]] lire.

Si l'on admet ces préalables, même en l'absence du mouvement, les constructions élidées de type (21), pourraient requérir une structure articulée :

(22) a. Je peux lire son roman, mais ses poèmes, je ne peux pas fop $_{\mathrm{i}} \mathrm{fes}_{\mathrm{i}}$ lire].

b. Je peux lire son roman, mais ses poèmes, je ne peux pas Eoples [poèmes]] lire.

Malheureusement, cette analyse se heurte à une autre difficulté : ce genre de structures est également possible avec des constructions classiquement vues comme impliquant un argument nul ${ }^{10}$, comme en (23) :

(23) J'ai porté tous les cartons, mais les meubles, j'ai refusé $\varnothing$ (sens : 'de les porter')

L'argument de Busquets et Denis nous semble donc difficile à maintenir, sinon sous réserve d'une analyse plus approfondie d'une part des propriétés exactes des proformes pleines et nulles vis-à-vis de ces constructions, et d'autre part du type exact de détachement ${ }^{11}$ à l'œuvre avec le 'pseudo-gapping à la française'. En revanche, d'autres arguments plaidant de façon moins problématique pour une lacune structurée peuvent être avancés pour compléter leur analyse. 


\subsection{Prolongements : mouvement-A- et mouvement WH-}

D'autres éléments, non mentionnés par ces auteurs, convergent en effet avec leurs conclusions. D'une part, ces modaux étant des verbes à montée du sujet, les vides doivent être structurés pour permettre la présence, notamment, de sujets d'inaccusatifs devant le modal, comme en. (24). Cette position est en effet classiquement attribuée à un mouvement-A depuis leur site d'insertion thématique, la position d'argument interne du V inaccusatif (v. Baltin : 2007 pour un traitement détaillé des inaccusatifs dans une structure elliptique) :

(24) a- Jean n'a pas pu venir mais heureusement [тр $\operatorname{Aude}_{\mathrm{i}}\left[\mathrm{a}\right.$ pu $<$ wenir $\left.\left.\mathrm{t}_{\mathrm{i}}>\right]\right]$. b- Jean n'a pas pu venir mais heureusement $\left[\mathrm{Tp}_{\mathrm{P}} \operatorname{Aude}_{\mathrm{i}}[\mathrm{a}\right.$ pu $\varnothing]$.

En (24b), avec une proforme non structurée, la source du sujet, 'Aude', demeure mystérieuse.

D'autre part, à côté des structures $\mathrm{ACD}$, qui impliquent un mouvement de l'opérateur ou de la tête, l'extraction de constituants WH- est également possible. C'est le cas notamment avec les relatives libres (cf. (25)) et, dans une certaine mesure, avec les interrogatives, (cf. (26)) :

(25) a- Il embrasse [wH qui i $_{\mathrm{i}}$ il peut $<$ embrasser ti>. b- Elle s'est attachée $\left[w_{w}\right.$ à qui $\left.i_{i}\right]$ elle $\mathrm{a} \mathrm{pu}<\mathrm{s}$ 'attacher ti $>$.

(26) a. Je sais quels livres Marie peut lire et quels livres elle ne peut pas. b. Je sais quels livres Marie peut lire et quels livres Ben ne peut pas. c. Je sais à qui je peux confier mon fils et à qui je ne peux pas.

La nécessité d'une position de base pour l'élément WH- explique l'agrammaticalité des phrases correspondantes impliquant une proforme pleine ${ }^{12}$ :

(27) a- *Il embrasse qui il le peut.

b- *Il embrasse qui il peut le faire.

(28) a- *Elle s'est attachée à qui elle l'a pu.

b- * Elle s'est attachée à qui elle a pu le faire.

(29) a- *Je sais quels livres Marie peut lire et quels livres elle ne le peut pas. b- *Je sais quels livres Marie peut lire et quels livres elle ne peut pas le faire.

(30) a- *Je sais quels livres Marie peut lire et quels livres Ben ne le peut pas. b- Je sais quels livres Marie peut lire et quels livres Ben ne peut pas le faire.

De même, les verbes permettant clairement un argument nul résistent à l'extraction WH- :

(31) a- *Jean a reçu qui Paul avait refusé $\varnothing$ (sens : 'de rencontrer')

b- *Jean a parlé à qui ses sœurs avaient refusé $\varnothing$ (sens : 'de parler').

Dans le cas des proformes, conçues comme non syntaxiquement structurées, ces faits s'expliquent aisément : le constituant déplacé ou coïndicé ne peut pas 'regarder à l'intérieur' de la proforme.

\subsection{Conclusion partielle}

La présence d'une lacune syntaxiquement structurée s'avère donc nécessaire pour rendre compte de divers processus syntaxiques affectant notamment les arguments directs, et disponibles avec les ellipses modales du français: la montée d'un opérateur relatif ou de la tête dans les constructions ACD, la montée d'un WH- avec les relatives libres et les interrogatives, la montée de l'argument interne des V inaccusatifs. Ces faits convergents trouvent une explication directe si l'on analyse les structures en jeu comme des structures elliptiques, alors qu'une analyse en proforme nulle peine à en rendre compte. En effet, dans ce cas, il faudrait postuler un fonctionnement différent des proformes vides et des proformes pleines, et de plusieurs types de proformes vides, ce qui saperait les arguments initialement avancés pour proposer une proforme vide. Seule l'analyse en ellipse des structures à modal permet de rendre compte de ces faits de façon cohérente. 


\section{L'ellipse modale est une ellipse du TP}

Notre proposition est la suivante: comme l'avancent Busquets et Denis (2001), l'ellipse modale est bien un cas d'ellipse. Mais en français, contrairement à l'anglais, il ne s'agit pas d'une ellipse du VP mais d'une ellipse du TP.

Cette proposition a au moins deux avantages. D'une part, elle est cohérente avec la généralisation couramment admise (Lobeck: 1995, Johnson: 2001, Merchant: 2001) sur la 'légitimation' (licensing) de l'ellipse : une tête autorise l'ellipse du constituant qu'elle sélectionne. Deuxièmement, elle offre une explication simple de l'agrammaticalité de certaines structures françaises qui sont possibles en anglais.

\subsection{Une ellipse du TP}

Rappelons tout d'abord que les modaux pouvoir et devoir, dans leurs emplois sans restructuration, sont communément considérés, pour les langues examinées ici, comme des verbes à montée du sujet, sélectionnant une proposition (Abeillé et Godard: 2003, Wurmbrand: 2000, Ruwet: 1972, Kayne: 1981) ${ }^{13}$. En cela, ils se distinguent de leurs équivalents anglais, qui sélectionnent une projection verbale. Nous considérons que la structure sélectionnée est du type illustré en (32):

$$
\text { (32) }[\mathrm{TP}[\text { ne pas [Asp [Aux-Passif [Voix [VP [VP ....]]]]] }
$$

La présence d'une projection Négation est due au fait que l'infinitif admet une négation autonome, cf. (33); celle d'une projection Aspect est due à la possibilité d'avoir un infinitif accompli (cf. (34)), ce qui, dans la lignée de Demirdache et Uribe-Etxebarria (2002), se traduit par la présence de l'auxiliaire avoir/être en AspP. A la suite de Collins (2005), nous considérons que la proposition héberge aussi une projection pour l'auxiliaire de passif et un syntagme Voix, puisque l'infinitif peut varier en voix, cf. (35).

(33) Elle ne peut pas ne pas fumer.

(34) Elle peut avoir lu ce livre pour jeudi.

(35) Ce meuble peut être soumis sans danger à d'importantes tractions.

L'hypothèse d'une ellipse du TP en français (vs une ellipse du VP en anglais), outre qu'elle respecte le principe que "ce qui est élidé est ce qui est sélectionné", permet de rendre compte des faits suivants: avec l'ellipse modale, aucun des éléments intervenant entre le modal et le VP ne peut être conservé, contrairement à ce qui se passe en anglais, comme l'illustrent les contrastes en (36)-(38) respectivement pour l'auxiliaire d'aspect, la négation, et l'auxiliaire du passif:

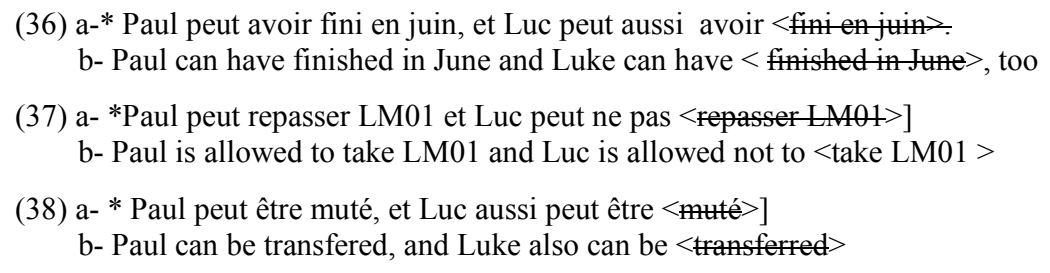

Ces contrastes s'expliquent très simplement si l'ellipse modale du français élide un TP, tandis que, comme le propose Merchant (2007) - contra Johnson (2007), Baltin (2007) - l'ellipse du VP anglaise élide effectivement le simple VP et non d'éventuelles projections verbales étendues.

\subsection{Asymétries de Voix}

En suivant plus loin Merchant (2007 et 2008), cette analyse devrait faire une prédiction qui semble, à première vue, vérifiée, même si un travail de corpus nous paraît nécessaire étant donné la subtilité des jugements. Merchant corrèle en effet la 'taille' de l'ellipse à la possibilité ou non d'avoir des asymétries de voix entre le constituant élidé et l'antécédent. Les ellipses 'hautes' (élidant plus que le simple VP), comme, pour l'anglais, le sluicing (ellipse du TP) ou le pseudo-gapping (ellipse du vP), n'autorisent pas 
les asymétries de voix, cf. (39), tandis que l'ellipse du VP, dans des conditions discursives adéquates, les permet, cf. (39'). Merchant attribue ce fait aux conditions d'identité que les constituants élidés doivent vérifier vis-à-vis de leurs antécédents: les ellipses hautes, sensibles à la présence du nœud Voix qu'elles incluent, exigent des traits de Voix identiques; en revanche, avec l'ellipse du VP, le nœud Voix est externe au site élidé, donc la Voix n'a aucune incidence sur les conditions d'identité.

(39) a. *Joe was murdered but I don't know who < murdered Joe $>$

b. *Someone murdered Joe but I don't know who by $<$ Joe was murdered $>$

(39') a. This problem was to have been looked into, but obviously nobody did .

b. The janitor should remove the trash whenever it is apparent that it needs to be

Si la proposition de Merchant est exacte et si l'ellipse modale française est une ellipse du TP, elle devrait donc exclure les asymétries de voix. Autant que nous puissions en juger, cela semble être le cas, comme en témoignent les exemples en (40), supposés remplir les conditions discursives les autorisant en anglais:

(40) a. *?Il faudrait remplacer l'ampoule ${ }_{\mathrm{i}}$ dans l'escalier, mais elle $_{\mathrm{i}}$ ne peut pas.

b. *? Ce problème aurait dû être résolu il y a longtemps, mais personne n'a pu.

Poser une ellipse du TP plutôt que du VP permet donc d'une part de prendre en compte la spécificité des modaux français en regard de leurs équivalents anglais, et de corréler cette différence à des différences empiriques dans les propriétés des ellipses associées aux modaux dans les deux langues. Dans la section suivante, nous montrons que faire de l'ellipse modale 'à la française' une ellipse du TP permet de rendre compte également d'une contrainte, passée jusque-là inaperçue, qui pèse sur certaines de ses occurrences et qui ne s'applique pas à l'ellipse du VP anglaise.

\section{La 'Contrainte des Sujets Similaires'}

\subsection{Les données du problème}

L'ellipse modale est disponible en français dans une série de contextes impliquant ou non une subordination, impliquant ou non un mouvement. Dans certains de ces contextes, néanmoins, elle est soumise à une contrainte particulière, que nous appelons 'Contrainte des Sujets Similaires' : le sujet placé devant le modal doit être coréférent à celui du VP antécédent. C'est le cas avec les constructions ACD et les relatives libres, comme l'illustrent respectivement en (41) et (42):

(41) a- Marie lit tous les livres qu'elle peut.

b- *Marie lit tous les livres que Jean ne peut pas/ peut.

(42) a- Jean embrasse qui il peut.

b- *Jean embrasse qui son frère ne peut pas / peut.

En revanche, elle ne s'applique pas, à première vue, aux constructions coordonnées, cf. (43), ni aux subordonnées n'impliquant pas de mouvement, cf. (44) :

(43) Jean peut parler à Pierre et Marie peut aussi.

(44) a- Paul viendra et Marie pense que Paul pourra, également.

b- Paul viendra dès qu'il pourra / Paul est venu parce que Marie ne pouvait pas

c- Paul viendra mais Marie ne sait pas si Luc pourra

$\mathrm{Ni}$ aux interrogatives, qui impliquent un mouvement WH- :

(45) Je sais quels livres Marie peut lire et quels livres Ben ne peut pas.. ${ }^{14}$

Les analyses en proformes nulles proposées pour l'espagnol, l'italien et le néerlandais (Depiante : 2001, Cecchetto et Percus: 2006, Aelbrecht: 2007) s'appuient principalement sur l'impossibilité des constructions ACD pour avancer que les lacunes suivant les modaux ne permettent pas d'extraction, et sont par conséquent des proformes nulles. Mais les exemples sur lesquels repose cet argument présentent tous des sujets disjoints, comme en (46)-(47) : 
(46) *Potrei mangiare ogni pizza OP che Gianni potrebbe (C\&OP : 29b) 'Je peux manger chaque pizza que Gianni peut'

(47) *Joris leest elk boek dat Monika moet (Aelbrecht: 2007) Joris lit chaque livre que Monika doit

Or, dans ces langues également, la coréférence des sujets autorise les constructions $\mathrm{ACD}^{15}$ :
(48) a. Maria legge tutti i libri che puó
[Italien]
b. María lee todos los libros qué puede [Espagnol]
c. Joris leest elk boek dat hij kan [Néerlandais ; L. Aelbrecht, p.c.]

Au vu de ces données, le contrainte des sujets similaires n'apparaît donc ni spécifique au français (cf. 48), ni spécifique à l'ellipse modale dans son ensemble (cf. 43-45). Dans la mesure où elle n'apparaît clairement en français que dans les structures à relativisation, il pourrait s'agir d'une contrainte uniquement liée à la relativisation même. Mais deux arguments vont d'emblée à l'encontre de cette explication.

D'une part, les structures non élidées ne sont soumises à aucune contrainte de ce type, comme le montre le contraste en (49) :

(49) a- *Marie lit tous les livres que Jean ne peut pas.

b- Marie lit tous les livres que Jean ne peut pas lire.

D'autre part, elle ne s'applique pas à l'anglais, où, contrairement au français, si un contexte plausible est disponible, des sujets disjoints sont possibles, cf. (50-51), vs, pour le même contexte, (50') et (51') :

(50) [ John is married to Mary, and he is blind: he cannot read; So, Mary reads aloud for him; In fact,] Mary reads every book that John can’t. (J. Merchant, p.c.)

(51) [John is Lea's father. Lea is 6 year old, and she is learning to read. Every evening, they read a story before Lea goes to bed. In fact, she reads and he occasionally helps her :] John reads every word that Lea can't. (J. Runner, p.c.)

(50') [Jean est marié à Marie, et il est aveugle : il ne peut pas lire. Alors, tous les soirs, Marie lui fait la lecture à haute voix. [ En fait, ] * Marie lit tous les livres que Jean ne peut pas.

(51') [Jean est le père de Léa, qui a 6 ans et apprend à lire. Tous les soirs, ils lisent une histoire avant que Léa aille se coucher. En fait, maintenant, c'est Léa qui lit et il l'aide un peu] * Jean lit tous les mots que Léa ne peut pas.

Notre hypothèse est que cette contrainte découle d'une part de propriétés propres aux structures $\mathrm{ACD}^{16}$, et de l'autre, d'une propriété commune aux verbes modaux du français, de l'espagnol, de l'italien et du néerlandais, qui les distingue des modaux anglais : ce ne sont pas des auxiliaires, mais des $\mathrm{V}$ à montée du sujet suivis d'une structure propositionnelle.

\subsection{La construction ACD}

La structure exacte des constructions ACD fait l'objet de débats rarement interrompus depuis les années 1970. S'il existe un consensus, au sein des approches génératives, pour considérer qu'elle implique une ellipse, diverses propositions ont tenté de résoudre les difficultés qu'elle pose. La première difficulté est que cette ellipse repose sur un apparent paradoxe syntaxique: le VP antécédent semble contenir le VP élidé; si le VP élidé est conçu comme un reflet syntaxique de son antécédent, ce qui est généralement considéré comme une condition d'identité légitimant l'ellipse, on aboutit à une régression infinie, cf. (52):

(52) Polly visited every country Erik $\operatorname{did}<$ visit every country Erik did $<$ visit .....>>.

Pour résoudre ce problème, il a été proposé - sous diverses formes à la suite de la proposition initiale de Sag (1976) - que le DP contenant la relative soit déplacé par mouvement (généralement, un avatar de “Quantifier Raising”) hors du VP antécédent, donnant un équivalent de (53), qui permet de restituer des structures parallèles pour le VP élidé et le VP antécédent (read $t)$ : 
Cette analyse se heurte néanmoins à une série de difficultés, relevant notamment de plusieurs faits de liage (Fox: 2002) et de contraintes de parallélisme encore relativement mal comprises entre le site élidé et l'antécédent (Kennedy: 1994, Sauerland: 2000, 2004). En particulier, Sauerland note que les analyses précédentes devraient prédire (à tort) comme bonnes des phrases élidées telles que (54):

(54) a. Polly visited every town that's near the one Eric did. [Sauerland 2000: 24a]

b. *Polly visited every town that's near the lake Eric did. [Sauerland 2000: 24b]

Afin d'en rendre compte, il propose une analyse qui repose sur la théorie du mouvement par copie, et adopte, pour les relatives à $\mathrm{ACD}$, en raison de faits de liage, une analyse de type "matching" correspondant, grossièrement ${ }^{17}$, à la structure en (55), où le NP interne est ensuite effacé:

\section{(55) the lake [Op 'lake'] Erik visited t].}

Dans une représentation simplifiée, tenant compte du mouvement du sujet interne de sa position thématique à l'intérieur de la projection verbale vers sa position en spec, $\mathrm{CP}$, on a donc pour l'anglais la représentation suivante :

(56) John reads every word [CP $\operatorname{word}_{2}$ that Lea 1 can’t [vP Lea 1 [vP read [the word 2$\left.\left.]\right]\right]$

L'ellipse efface le VP, ce que nous matérialisons ci-dessous par une biffure double, la copie du sujet interne étant indépendamment effacée à la suite du mouvement, et le NP interne à la relative par vérification avec l'antécédent :

(56') John reads every word [CP word $_{z}$ that Lea ${ }_{1}$ can't $\left[{ }_{\mathrm{vP}}\right.$ Lea $\left.\left._{4}[\mathrm{vP}]\right]\right]$

Les constituants élidés doivent respecter des contraintes d'identité sémantico-syntaxique que nous ne détaillerons pas ici faute de place, ce qui explique la bonne formation de $(54 \mathrm{a})$, où the one et the town remplissent les conditions adéquates, et l'agrammaticalité de $(54 \mathrm{~b} / 57)$, dans la mesure où [visit town] et [visit lake] violent ces conditions d'identité :

\section{(57) *Polly visited every town that's near the lake [CP take that Erik did [vP Erik [WP [isithe [ake]}

Nous proposons ici que la contrainte des sujets similaires, qui ne s'applique pas en anglais mais intervient en français et dans les autres langues citées, trouve une explication dans ce cadre, si l'on tient compte des spécificités respectives des modaux dans les deux types de langues.

En effet, en anglais, l'auxiliaire sélectionne une projection du verbe, qui, dans l'ellipse du VP, est élidée. C'est ce constituant qui doit vérifier les conditions d'identité avec son antécédent. En (51/56), c'est donc, selon l'analyse de Merchant (2007), le VP read the word: La seule contrainte porte donc d'une part sur le verbe, et d'autre part sur l'argument relativisé.

En revanche, en français et dans les autres langues soumises à la contraintes des sujets similaires, le modal sélectionne un TP, et c'est ce TP qui est élidé. La représentation contenant les copies de mouvement est donc la suivante (nous ne notons que les projections et mouvements pertinents):

(58) *Jean lit tous les mots [CP mots que Lea $_{1}$ ne peut pas

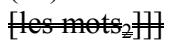

Le constituant élidé est soumis de la même manière aux conditions d'identité. Mais cette fois, le constituant élidé contient une copie du sujet, qui doit de ce fait être sémantiquement identique à celui de la phrase antécédente. Quand c'est le cas, comme en (59), la phrase est grammaticale. Quand ce n'est pas le cas, comme en (58), la violation des conditions d'identité rend la phrase agrammaticale.

(59) $\mathrm{Jean}_{1}$ lit tous les mots [CP mots 2 que il peut [1]

La taille de l'ellipse, corrélée au statut des modaux, rend donc compte du fait que la contrainte des sujets similaires intervient en français, en italien, en espagnol et en néerlandais, mais pas en anglais. Cette 
contrainte n'est qu'un avatar de la contrainte d'identité, appliqué à la structure particulière sélectionnée (et donc éventuellement élidée) par les modaux.

\subsection{Problèmes en suspens}

Si cette analyse rend compte d'une large palette de propriétés associées à l'ellipse modale en français, un certain nombre de problèmes qu'elle soulève restent à résoudre.

D'une part, il est communément admis que les éléments focalisés échappent aux conditions d'identité (voir Merchant: 2001, par exemple, pour une proposition d'analyse). Or, dans les constructions ACD du français, les sujets ne semblent pas pouvoir y échapper. Plusieurs pistes d'explication sont possibles, qu'il reste à explorer. Dans la mesure où les sujets peuvent, en français, être disjoints dans les autres constructions impliquant une ellipse modale (coordinations, autres subordonnées), il est possible que le marquage du focus dans les relatives restrictives soit en cause, ce qui serait sans incidence pour l'anglais puisque les sujets ne sont pas inclus dans le site élidé. C'est la voie explorée par Dagnac (2008), qui montre que certaines constructions focalisées, comme la dislocation ou le focus étroit sur un DP sujet, possibles ailleurs, sont effectivement impossibles dans une restrictive relative non élidée, ce qui empêche la relative élidée d'échapper à la contrainte des sujets similaires par ce biais. Mais l'idée qu'aucun procédé de focalisation ne soit disponible dans ces relatives semble trop stricte. Il est donc également possible que le type exact de focus permettant la levée de l'identité stricte ne soit pas disponible dans les relatives restrictives, ou plus généralement en français, ou encore dans les seules relatives restrictives françaises. Pour trancher entre ces possibilités, il reste d'une part à comparer les latitudes de focalisation dans les diverses langues permettant l'ellipse modale dans les constructions ACD et d'autre part à affiner les modalités de focus contrastif et leur impact exact sur les structures elliptiques.

Ce dernier point paraît indépendamment nécessaire au regard du fait suivant. En anglais, il existe également deux cas où le focus contrastif ne parvient pas à assouplir la condition d'identité : Sauerland (2000, note 31) souligne en effet, sans pouvoir résoudre la question, que le jeu du focus devrait prédire que (60) peut être grammaticale avec un accent de focus sur le syntagme de town, puisque lake est une alternative focale pour town. ${ }^{18}$ Or, ce n'est pas le cas (les constituants focalisés sont notés F) :

(59) a. *Do you know which town near a lake Mary visited John did $<$ visit $t>$

b. which $[\text { town }]_{\mathrm{F}}$ near a lake $\lambda \mathrm{y}$ Mary visited $[\mathrm{y}$, lake $\left.]\right] \lambda \mathrm{x}$ John did visit $\left.[\mathrm{x}, \text { town }]_{\mathrm{F}}\right]$

Zoerner et Agbayani (2002) remarquent également une contrainte d'identité des sujets, mais affectant le pseudo-gapping anglais dont le constituant maintenu est autre qu'un DP, cf. (61) :

(61) a. * You probably just feel relieved, but I do feel jubilant

b. I don't feel jubilant, but I do feel relieved

Ce fait est intéressant car, si l'on suit toujours Merchant (2007, 2008), le pseudo-gapping anglais implique une ellipse du vP, donc, prima facie, élide un constituant contenant, comme les ellipses modales, une copie du sujet.

Enfin, la solution proposée repose crucialement sur un traitement uniforme des copies de mouvement-A et de mouvement-A', puisque la montée du sujet est considérée ici comme un mouvement-A. En cela, nous nous écartons de Sauerland (2002, 2004) - vs, par exemple, Kennedy (1994), Heim (1997). L'incidence de ce choix dans le débat en cours sur le statut des copies reste à évaluer.

\section{Conclusion}

Une large palette de faits d'extraction (mouvement de l'opérateur/de la tête, mouvement A, mouvement WH-) est compatible avec l'ellipse modale en français, ce qui argumente fortement en faveur d'une analyse posant, après le modal, une structure syntaxique pleinement articulée mais non instanciée lors du traitement phonologique. Néanmoins, ces structures présentent des différences avec l'ellipse du VP classique de l'anglais. L'hypothèse qu'il s'agit d'une ellipse du TP, et non du VP permet, selon nous, de 
rendre compte de ces différences de façon directe, notamment de l'impossibilité de maintenir après le modal des constituants placés au-dessus du VP, tout en corrélant ces propriétés aux propriétés sélectionnelles des modaux dans les deux langues. Elle a par ailleurs l'avantage de ne pas modifier l'appartenance typologique du français aux langues 'sans ellipse du VP' Si l'analyse que fait Merchant (2007) des asymétries de voix est exacte, et si les premières données correspondantes du français se confirment, elle permet aussi d'expliquer que l'ellipse modale ne se comporte pas, de ce point de vue, comme son équivalente anglaise. Par ailleurs, nous avons fait apparaitre que les ellipses modales associées à une relativisation sont soumises à une contrainte particulière, la 'Contrainte des Sujets Similaires', qui pour l'instant n'a pas été prise en compte dans les analyses de ce phénomène. Cette contrainte, si elle est absente de l'anglais, pèse sur les constructions semblables de l'espagnol, de l'italien et du néerlandais, pour lesquels les modaux, dans leur version non restructurante, ont un comportement très similaire à celui du français. Si cette contrainte est vérifiée, des extractions hors du site lacunaire sont possibles dans ces langues aussi, ce qui fragilise les analyses en proforme nulle précédemment avancées à leur sujet. Nous proposons que cette contrainte soit directement corrélée à la structure du constituant élidé : dans la lignée de Sauerland (2000,2004), nous considérons que les copies de mouvement présentes dans le site élidée sont soumises à des conditions d'identité. Dans notre analyse, avec les modaux à montée, la copie du sujet est présente dans le TP élidé et donc soumise à ces conditions d'identité. La nature exacte de ces conditions fait l'objet de nombreux débats, auxquels ces nouvelles données apportent un éclairage intéressant. Enfin, le fait qu'un focus contrastif puisse assouplir ces conditions dans les structures sans relativisation apporte une nouvelle pierre au chantier en cours sur l'étude de l'interaction du focus, de l'ellipse et du mouvement.

\section{Références}

Abeillé A., Godard D. (2003). Les Prédicats complexes dans les langues romanes. In Godard, D. (ed), Les langues romanes. Paris : CNRS Editions, 125-184.

Aelbrecht, L. 2007. Dutch modals with null proform VP complements. Communication au CSSP (Colloque de Syntaxe et Sémantique de Paris), 4-6 Octobre 2007.

Baltin, M. (2007). Deletion Versus Pro-Forms: A False Dichotomy? Ms, New York University.

Bianchi V. (1995). Consequences of Antisymmetry for the syntax of headed relative clauses. Thèse de doctorat. Scuola Normale Superiore, Pisa.

Busquets, J., Denis, P. (2001). L'Ellipse modale en français. Cahiers de Grammaire 26, 55-74.

Cecchetto, C., Percus, O. (2006). When we do that and When we don't: a contrastive analysis of VP ellipsis and VP anaphora. In M. Frascarelli (ed), Phases of Interpretation, Mouton de Gruyter, 71-105.

Chung S., Ladusaw W., McCloskey J. (1995). Sluicing and Logical Form. Natural Language Semantics 3, 239-282.

Cinque G. (2007). The prenominal origin of relative clauses. Communication au CSSP, ENS, Paris, 4-6 octobre 2007.

Collins, C. (2005). A Smuggling Approach to the Passive in English. Syntax 8: 2, 81-120.

Dagnac, A. (2008), French, Modal Ellipsis and the 'Same Subject Constraint', Communication à LSRL 38, University of Illinois at Urbana-Champaign, 4-6 avril 2008.

Depiante, M. (2001). On Null Complement Anaphora in Spanish and Italian. Probus 13, 193-221

Delais-Roussarie E. et al.. (2004). Dislocation. In F. Corblin et H. de Swart (eds), Handbook of French Semantics. Stanford: CLSI, 501-528.

Demirdache H., Uribe-Etxebarria, M..(2002). La Grammaire des prédicats spatiotemporels: temps, aspect et averbes de temps. In B. Laca (ed), Temps et Aspect. Paris : Presses Universitaires de Vincennes, 125-176.

Fiengo, R., May, R. (1994). Indices and Identity. Cambridge : MIT Press.

Fox, D. , Lasnik, H.. (2003). Successive Cyclic Movement and Island Repair. Linguistic Inquiry 34 :1, 143-154.

Fox, D. (2002). ACD and the Copy Theory of Movement. Linguistic Inquiry $33: 1$, 63-96. 
Gengel K. (2007). Phases and Ellipsis. In E. Efner, and M. Walkow (eds.). Proceedings of the 37th meeting of the North East Linguistic Society. University of Massachusetts, Amherst : GLSA Publications.

Guilliot N. (2006). La Recontruction à l'interface entre syntaxe et sémantique: ou comment interpréter les copies syntaxiques. Thèse de Doctorat, Université de Nantes.

Hankamer, J., Sag I. (1976). Deep and Surface Anaphora. Linguistic Inquiry 7 : 3, 391-428.

Hardt, D. (1999). Dynamic Interpretation of Verb Phrase Ellispsis. Linguistics and Philosophy 10, 503-530.

Heim I. (1997). Predicates or Formulas? Evidence from Ellipsis. In Lawson A. et Cho E. (eds), Proceedings of SAALT 7. Ithaca, New York : CLC Publicatins.

Johnson, K.. (2001). What VP Ellipsis Can Do, and What it Can't, but not Why. In M. Baltin and C. Collins (eds), The Handbook of Contemporary Syntactic Theory, Blackwell Publishing, 439-479.

Johnson, K.. (2004). How To Be Quiet. Communication à CLS 40. http://people.umass.edu/kbj/homepage.

Kayne R. (1994). The Antisymmetry of syntax. Cambridge : MIT Press.

Kennedy, C. (1994). Argument Contained Ellipsis. UC Santa Cruz : Linguistics Research Center Report LCR 94-03.

Lobeck, A. (1995). Ellipsis: Functional Heads, Licensing and Identification. Oxford : Oxford University Press.

Merchant, J. (2001). The Syntax of Silence. Oxford : Oxford University Press.

Merchant, J. (2001b/2008). Variable Island Repair under Ellipsis. A paraître dans K. Johnson (ed), Topics in Ellipsis, Cambridge University Press.

Merchant J. (2006). Syntactic abstractness in ellipsis: sluicing, wh-movement, islands. Ealing 2006, Paris, ENS.

Merchant, J. (2007). Voice and Ellipsis. Ms, University of Chicago.

Merchant, J. (2008). An asymmetry in voice mismatches in VP-ellipsis and pseudogapping. Linguistic Inquiry $39,169-179$.

Rooryck, J. (2000). Configurations of Sentential Complementation. Londres: Routledge.

Ruwet, N. (1972). Théorie syntaxique et syntaxe du français. Paris: Seuil.

Sauerland U. (2000). Obligatory reconstruction and the meaning of traces. Arbeitspapiere des SFB 340, Nr 153, 1-28.

Sauerland U. (2004). The Interpretation of Traces. Natural Language Semantics 12, 63-127.

Shopen, T. (1972). A Generative Theory of Ellipsis : A consideration of the linguistic use of silence. Reproduit par le Indiana University Linguistics Club.

Vergnaud J.R. (1973). French relative clauses. PhD Thesis, MIT.

Wurmbrand, S. (2007). Infinitives are Tenseless. A paraître dans U. Penn Working Papers in Linguistics.

Wurmbrand, S. (2001). Infinitives : Restructuring and Clause Structure. Berlin : Mouton De Gruyter.

Wurmbrand, S. (1999). Modal Verbs must be raising verbs. In S. Bird et al. (eds), WCCFL 18 Proceedings. Sommerville, MA: Cascadilla Press, 599-612.

Zoerner E. \& B. Agbayani. 2002. A Pseudogapping asymmetry. Snippets 5: 18-19.

\footnotetext{
${ }^{1}$ Ces structures ont également fait l'objet de débat dans d'autres cadres théoriques, que nous n'aborderons pas ici.

${ }^{2}$ La nature de ce parallélisme et les rôles respectifs qu'y jouent syntaxe et sémantique, est elle-même objet de débats.

${ }^{3}$ Pour certaines (Espagnol, Italien), ils peuvent être en outre restructurants, ce qui se manifeste avant tout par le montée optionnelle des clitiques (Abeillé et Godard : 2003, inter alia) : dans la version restructurée, les clitiques correspondant aux arguments sélectionnés par le verbe infinitif se placent sur le modal, cf. (i). Cette option n'est plus disponible en français contemporain courant (ii), et les structures elliptiques illustrées en (6-7) semblent, pour l'italien et l'espagnol, limitées à la version non restructurée des constructions modales (Depiante :2001).
}

(i) Lo voglio dare a Lucia. [Italien] 
${ }^{4}$ Par commodité, nous notons pour l'instant les positions initiales par des traces, sans préjuger de leur nature. Pour une analyse des traces comme copies, voir par exemple Fox (2002), Sauerland (2002, 2004), et la section 4.

${ }^{5}$ Nous ne tenons pas compte pour l'instant de l'idée que l'opérateur peut avoir la structure [Op, N], ni de la théorie du mouvement par copie. Voir la section 4.

${ }^{6}$ Une précision terminologique s'impose: l'élément déplacé est un 'topic' ('thème'), qui porte une marque de focus (contrastif) car il entre dans une rapport de contraste avec le constituant équivalent présent dans l'antécédent. Par ailleurs, l'exigence d'un mouvement ouvert en français mais pas en anglais reste à expliquer. Une différence paramétrique liée au statut du marquage accentuel-intonatif dans les deux langues semble plausible.

${ }^{7}$ De ce point de vue, il nous semble que (i) est acceptable, alors que (ii) nous semble beaucoup plus difficile :

(i) Personne de mon entourage ne peut lire Minute. Mais le Figaro, je connais plusieurs personnes qui peuvent.

(ii) ??Personne ne peut dire la vérité à Jim. Mais à Jules, je connais beaucoup de gens qui pourraient.

${ }^{8}$ Voir néanmoins Guilliot (2006) pour une synthèse des problèmes que pose cette analyse.

${ }^{9}$ Pour une argumentation détaillée, voir Guillot (2006).

${ }^{10}$ Cette analyse est confirmée par le fait qu'elles ne permettent pas les autres types d'extraction examinés ici, cf. (i) :

(i) *J'ai finalement porté tous les cartons que dans un premier temps j'avais refusé.

${ }^{11}$ Ceci mérite une étude spécifique, tenant compte des contextes. En français, pour Delais-Roussarie et al. (2004), une différence majeure entre dislocation et topicalisation est leur aptitude ou non à remodeler le Discourse Topic.

${ }^{12}$ Cette affirmation doit être nuancée: elle s'applique strictement aux extractions d'arguments directs; en revanche, certains arguments indirects et adjoints peuvent être extraits malgré la présence d'une proforme pleine. Ce point ne nous semble pas remettre en question le principe de base, mais plutôt relever des propriétés de ces proformes, qui fonctionnent comme des pro-VP, pouvant reprendre le VP minimal (V + argument direct) ou élargi. C'est donc l'accessibilité de l'argument direct qui, stricto sensu, est cruciale ici.

${ }^{13}$ Pour d'autres propositions, voir notamment Wurmbrand (2007) et Rooryck (2000).

${ }^{14}$ L'extraction d'un interrogatif est soumis, comme pour l'ellipse de VP anglaise (Fox et Lasnik: 2003, Merchant: 2001b) voire au-delà, à des contraintes de parallelisme et de contraste fortes, que nous ne pouvons traiter ici.

${ }^{15}$ Ces données sont problématiques pour les analyses en proforme nulle qui ont été proposées pour ces langues. Pour d'autres arguments en faveur d'une ellipse modale en espagnol et en italien, voir Dagnac (2008).

${ }^{16}$ Nous laissons pour l'instant de côté les relatives libres, dont le traitement, en raison des problèmes spécifiques qu'elles posent, excèderait largement le cadre de cette étude. Il ne semble pas absurde d'y voir un cas particulier d'ACD, dans la mesure où elles contiennent une quantification implicite.

${ }^{17}$ Pour la relation exacte entre le NP antécédent et le NP interne à la relative, voir Sauerland (2004).

18 « Since the elided VP contains a trace of wh-movement in (ia), focus percolation from the wh-phrase should be possible, and the focus structure in (ib) should result. But, then (ia) should be acceptable with focus on town-phrase, since lake is a focus alternative to town that would satisfy direct parallelism. This prediction seems factually incorrect. [...] While I suspect that a better understanding of what are possible focus structures will provide an explanation for (ia), at this point, I have to leave the matter open. » 